BMJ Open Diabetes Research \& Care

\title{
Physical behaviors and chronotype in people with type 2 diabetes
}

\author{
Joseph Henson (D) , ${ }^{1}$ Alex V Rowlands, ${ }^{1,2}$ Emma Baldry (iD , ${ }^{3}$ Emer M Brady, ${ }^{4}$ \\ Melanie J Davies, ${ }^{1,3}$ Charlotte L Edwardson, ${ }^{1}$ Thomas Yates, ${ }^{1}$ Andrew P Hall, ${ }^{5}$ \\ CODEC Investigators
}

To cite: Henson J, Rowlands AV, Baldry E, et al. Physical behaviors and chronotype in people with type 2 diabetes. BMJ Open Diab Res Care 2020;8:e001375. doi:10.1136/ bmjdrc-2020-001375

\section{- Additional material is published online only. To view please visit the journal online (http://dx.doi.org/10.1136/ bmjdrc-2020-001375).}

Received 17 March 2020 Revised 2 June 2020 Accepted 10 June 2020

\section{Check for updates}

(C) Author(s) (or their employer(s)) 2020. Re-use permitted under CC BY-NC. No commercial re-use. See rights and permissions. Published by BMJ.

${ }^{1}$ NIHR Leicester Biomedical Research Centre and Diabetes Research Centre, College of Life Sciences, University of Leicester, Leicester, UK ${ }^{2}$ Alliance for Research in Exercise, Nutrition and Activity (ARENA), Sansom Institute for Health Research, University of South Australia Division of Health Sciences, Adelaide, South Australia, Australia ${ }^{3}$ Diabetes Research Centre, University Hospitals of Leicester NHS Trust, Leicester, UK ${ }^{4}$ Department of Cardiovascular Sciences, University of Leicester, Leicester, UK ${ }^{5}$ Hanning Sleep Laboratory, University Hospitals of Leicester NHS Trust, Leicester, UK

Correspondence to Dr Joseph Henson; jjh18@le.ac.uk

\section{ABSTRACT}

Introduction Previous investigations have suggested that evening chronotypes may be more susceptible to obesityrelated metabolic alterations. However, whether devicemeasured physical behaviors differ by chronotype in those with type 2 diabetes (T2DM) remains unknown.

Research design and methods This analysis reports data from the ongoing Chronotype of Patients with Type 2 Diabetes and Effect on Glycaemic Control (CODEC) observational study. Eligible participants were recruited from both primary and secondary care settings in the Midlands area, UK. Participants were asked to wear an accelerometer (GENEActiv, Activlnsights, Kimbolton, UK) on their non-dominant wrist for 7 days to quantify different physical behaviors (sleep, sedentary, light, moderate-to-vigorous physical activity (MVPA), intensity gradient, average acceleration and the acceleration above which the most active continuous 2, 10, 30 and $60 \mathrm{~min}$ are accumulated). Chronotype preference (morning, intermediate or evening) was assessed using the Morningness-Eveningness Questionnaire. Multiple linear regression analyses assessed whether chronotype preference was associated with physical behaviors and their timing. Evening chronotypes were considered as the reference group.

Results 635 participants were included (age $=63.8 \pm 8.4$ years, $34.6 \%$ female, body mass index $\left.=30.9 \pm 5.1 \mathrm{~kg} / \mathrm{m}^{2}\right)$. $25 \%(n=159)$ of the cohort were morning chronotypes, $52 \%(n=330)$ intermediate and $23 \%(n=146)$ evening chronotypes. Evening chronotypes had higher sedentary time (28.7 $\mathrm{min} /$ day, $95 \% \mathrm{Cl} 8.6$ to 48.3$)$ and lower MVPA levels (-9.7 min/day, -14.9 to -4.6$)$ compared to morning chronotypes. The intensity of the most active continuous 2-60 min of the day, average acceleration and intensity gradient were lower in evening chronotypes. The timing of physical behaviors also differed across chronotypes, with evening chronotypes displaying a later sleep onset and consistently later physical activity time.

Conclusions People with T2DM lead a lifestyle characterized by sedentary behaviors and insufficient MVPA. This may be exacerbated in those with a preference for 'eveningness' (ie, go to bed late and get up late).

\section{INTRODUCTION}

Type 2 diabetes (T2DM) is a condition characterized by hyperglycemia, resulting from defects in hepatic and peripheral glucose uptake, insulin secretion or both. ${ }^{1}$ In 2019 , the global prevalence of T2DM in 20-79 year

\section{Significance of this study}

What is already known about this subject?

- Quantity of sleep is associated ('U' shaped relationship) with the development of cardiometabolic disease. Beyond duration of sleep, the timing of sleep may also be important.

- Chronotype, a diurnal characteristic, identifies individuals as having a preference for morning (ie, go to bed early and get up early), evening (ie, go to bed late and get up late) or neither (intermediate).

- Evening chronotypes may be more susceptible to obesity-related metabolic alterations, potentially driven by differences in physical behaviors.

- Adults with type 2 diabetes (T2DM) are less physically active and more sedentary than those without the condition.

\section{What are the new findings?}

- Our study demonstrates that those with a preference for an evening chronotype have higher sedentary time, lower levels of light activity and lower moderate-tovigorous physical activity (MVPA) compared with both morning and intermediate chronotypes.

- The timing of both sleep onset and physical activity differs across chronotypes, with evening chronotypes displaying a later sleep onset and consistently later physical activity time.

- Average MVPA levels were $56 \%$ lower in evening chronotypes compared with morning chronotypes.

- In addition to duration, differences also exist in the intensity of movement between chronotypes.

\section{How might these results change the focus of} research or clinical practice?

- There is a need for large-scale interventions to be implemented into diabetes care which support people with T2DM to initiate, maintain and achieve the substantial benefits of an active lifestyle. This may be particular pertinent for those with an 'eveningness' preference.

- As chronotype is potentially modifiable, future research should focus on these physical behaviors (and their timing) in order to optimize interventional responses.

olds was estimated to be 463 million (11\%), with this figure expected to rise further to 700 million by $2040 .^{2}$ Over the last three decades, the number of people with diabetes has more 
than doubled and it is the fifth leading cause of mortality globally. ${ }^{3}$

Healthy sleep is considered an important lifestyle component that is associated with chronic disease. ${ }^{4}$ For example, an increasing number of studies have demonstrated that the quantity of sleep is associated ('U' shaped relationship) with the development of cardiometabolic disease including metabolic syndrome, T2DM and cardiovascular disease ${ }^{56}$ Beyond duration of sleep, the timing of sleep may also be important. Chronotype, a diurnal characteristic that identifies individuals as having a preference for morning (ie, go to bed early and get up early), evening (ie, go to bed late and get up late) or neither (intermediate), is driven by endogenous circadian rhythms, environmental (eg, light), sociooccupational (eg, employment) and lifestyle factors. ${ }^{78}$ Previous investigations have demonstrated that evening chronotypes may be more susceptible to obesity-related metabolic alterations. ${ }^{9}{ }^{10}$ However, in order to inform future lifestyle interventions, it is necessary to explore the role of potentially modifiable determinants (eg, sedentary behavior and physical activity) across different chronotypes.

Regular physical activity is an important lifestyle recommendation for the management of T2DM that is associated with substantially lower cardiovascular and overall mortality risk. ${ }^{11}{ }^{12}$ Individuals are encouraged to exercise daily or at least every other day, ${ }^{12}$ with the aim of meeting the current physical activity guidelines of at least $150 \mathrm{~min}$ a week of moderate physical activity or $75 \mathrm{~min}$ of vigorous intensity exercise per week. ${ }^{13}$ Reducing the amount of time spent sedentary is also recommended for the management of T2DM. ${ }^{12}$ Despite the unequivocal benefits of moderate-to-vigorous physical activity (MVPA) and deleterious effects of sedentary behavior, adults with T2DM are less physically active and more sedentary than those without the condition. ${ }^{14-16}$ The advancement of technology (such as accelerometry) now allows devicemeasured physical behaviors to be quantified continuously over a period of time (including the ability to timestamp physical behaviors), while addressing many of the limitations previously associated with self-report (eg, reporting bias). They also offer the opportunity to quantify sleep, which is a complex and multidimensional function that encompasses independent, but related, metrics including duration (quantity).

Despite previous investigations suggesting that those with a preference for an evening chronotype may engage in lower levels of physical activity, ${ }^{17}$ whether devicemeasured physical behaviors differ by chronotype in those with T2DM remains unknown. As chronotype is potentially modifiable, ${ }^{18}$ these common functions may be leveraged to individually tailor interventions targeting these behaviors to optimize responses. Therefore, the aim of this manuscript is to describe physical behaviors (including their timing) in a multiethnic population with T2DM and examine whether these differ by chronotype preference.

\section{RESEARCH DESIGN AND METHODS}

\section{Participants and methods}

Participants included in this analysis had data collected as part of the Chronotype of Patients with Type 2 Diabetes and Effect on Glycaemic Control (CODEC) study. Briefly, this is a cross-sectional, multisite observational study which primarily aims to investigate and describe the chronotype of patients with T2DM and the subsequent effect on glycemic control. ${ }^{19}$ Secondary aims include exploring the associations between glycemic control, cardiometabolic health and other lifestyle factors (including sedentary and physical activity monitoring). Detailed inclusion and exclusion criteria are presented in online supplementary table 1.

Eligible participants were recruited from both primary and secondary care settings from four sites across the Midlands, UK (Leicester, Nottingham, Derby, Northampton). All participants provided written informed consent.

Outcomes of interest were collected following informed consent at the data collection appointment. These included date of birth, sex (male/female), ethnicity (self-reported) and body mass index (BMI) $\left(\mathrm{kg} / \mathrm{m}^{2}\right)$. BMI was calculated to the nearest $0.1 \mathrm{~kg} / \mathrm{m}^{2}$. Medical history (including duration of T2DM) was collected by a study clinician, research nurse or healthcare professional. Occupation type was also collected via questionnaire (self-report).

\section{Chronotype}

Chronotype preference was determined using the Morningness-Eveningness self-assessment questionnaire (MEQ) $)^{20}$ This validated questionnaire consists of 19 items on sleep habits and fatigue and assesses individual differences in the degree to which respondents are active and alert at certain times of day. The scale item responses determine preferences in sleep and waking times and subjective 'peak' times at which respondents feel their best. Individuals were classified as either evening type (score of $\leq 52$ ), intermediate type (53-64) or morning type $(\geq 65){ }^{21}$

\section{Physical behaviors: sleep, sedentary behavior and physical activity}

Participants were asked to wear an accelerometer (GENEActiv, ActivInsights, Kimbolton, UK) on their non-dominant wrist to quantify habitual levels of sleep, sedentary time and physical activity. The GENEActiv was initialized to collect data at $100 \mathrm{~Hz}$. Participants wore the GENEActiv accelerometer 24 hours/day for up to 7 days. Alongside this, participants also completed a wake and sleep log for the days they wore the device. The device was fitted on the day of their appointment and they were provided with a prepaid envelope to return the device and the wake/sleep $\log$ at the end of the assessment period.

\section{Accelerometer data processing}

On return, data were downloaded using GENEActiv PC software V.3.2. The GENEActiv.bin files were processed using R-package GGIR V.1.8-1 (http://cran.r-project.org) and a sleep detection algorithm was applied to derive sleep 
duration. ${ }^{22-25}$ Sleep logs were used to guide the algorithm to identify this sleep window.

Signal processing in GGIR includes autocalibration using local gravity as a reference, ${ }^{23}$ detection of sustained abnormally high values, detection of non-wear and calculation of the average magnitude of dynamic acceleration, corrected for gravity averaged over $5 \mathrm{~s}$ epochs and expressed in milligravitational units $(\mathrm{mg})$. Participants were excluded if their accelerometer files showed postcalibration error greater than $0.01 \mathrm{~g}(10 \mathrm{mg})$, fewer than 3 days of valid wear (defined as $>16$ hours per day ${ }^{22}{ }^{26}$ ) or wear data were not present for each 15 min period of the 24 hours cycle. Detection of non-wear has been described in detail previously. ${ }^{23}$ The default non-wear setting was used, that is, invalid data were imputed by the average at similar time-points on different days of the week. Sleep duration was calculated using automated sleep detection (HDCZA sleep detection algorithm). ${ }^{25}$ The average of all valid days was used for all outcome variables.

The following sedentary behavior and physical activity characteristics were obtained: average time accumulated in sedentary behavior (defined as time accumulated during the waking day below $40 \mathrm{mg}$ ), ${ }^{26}$ light activity (defined as time accumulated with an acceleration between 40 and $100 \mathrm{mg}$ ), ${ }^{27}$ MVPA in $1 \mathrm{~min}$ bouts (defined as time accumulated in $1 \mathrm{~min}$ bouts above an acceleration of $100 \mathrm{mg}$ ), ${ }^{28}$ average acceleration in $\mathrm{mg}$ (a proxy for overall physical activity) and the average acceleration of the most active continuous 2, 10, 30 and 60 min bouts). In addition, we also derived the time at which the MX metrics were undertaken, allowing analysis of when the most intensive periods of activity were occurring. MX is a novel accelerometer metric that captures the intensity (acceleration) during a person's most active period of the day. ${ }^{30}$

In addition, we report the intensity gradient, which reflects the distribution of physical activity intensity across the 24 hour day and has been described in detail elsewhere. ${ }^{31}$ In brief, it describes the negative curvilinear relationship between physical activity intensity and the time accumulated at that intensity during the 24 hour day. The intensity gradient is always negative, reflecting the drop in time accumulated as intensity increases; a more negative (lower) gradient reflects a lower amount of time accumulated at mid-range and higher intensities, while a less negative (higher) gradient reflects more time spread across the intensity range. ${ }^{31} \mathrm{MX}$ and the intensity gradient metrics do not rely on population-dependent and protocol-dependent cut-points to estimate intensity of physical activity.

The following sleep characteristic was obtained: sleep duration (total accumulated sleep within the sleep window), discounting any wake time and daytime sleep and the time of sleep onset.

\section{Statistical analysis}

Demographic, anthropometric, biochemical and accelerometer derived variables are presented as numbers (mean $(\mathrm{SD})$, median $(\mathrm{IQR})$ ) or percentages for categorical groups. All physical behaviors are reported as average minutes per day. Acceleration variables are reported as $\mathrm{m} g$.

Multiple linear regression analyses assessed whether time spent in physical behaviors or the timing of physical behaviors differed by chronotype preference, both unadjusted and independent of covariates (age, sex, ethnicity, employment status, duration of diabetes and sleep duration). Given the non-linear association between sleep and physical activity, sleep duration was split into tertiles before being entered into the linear model as a covariate. When examining the potential differences in the timing of physical behaviors, the continuous MX bouts were used.

A main effect of chronotype was followed by posthoc contrasts. Given that previous investigations have demonstrated a less favorable cardiometabolic profile in evening chronotypes, ${ }^{910}$ these individuals were considered as the reference group. Due to the right-skewed distributions of positive values, MVPA was analyzed using a gamma distribution with an identity link.

Interaction terms with chronotype were entered simultaneously into the same model to investigate whether the effect of chronotype was modified by sex, ethnicity, age, BMI or employment status independently to the other factors. All data were analyzed using SPSS (V.24.0). $\mathrm{P}<0.05$ was considered statistically significant for main effects and $\mathrm{p}<0.1$ for interactions. Results of the multiple linear regression are reported as mean (95\% CI).

\section{RESULTS}

\section{Participant characteristics}

Of the 808 currently enrolled onto the CODEC study, accelerometer files were available for 696 participants. Of these, 8 were excluded ( 4 technical issues, 4 insufficient wear) resulting in 688 with valid accelerometer data. Of these, 635 had valid MEQ and covariate data (age $63.8 \pm 8.4$ years, $34.6 \%$ female, BMI $=30.9 \pm 5.1 \mathrm{~kg} / \mathrm{m}^{2}$ ), which equated to $78.6 \%$ of the cohort. Those included in this analysis had a similar age, BMI, HbAlc, sex and ethnic breakdown compared with those who did not reach the minimum accelerometer criteria or those who did not complete the MEQ.

Table 1 displays the demographic, anthropometric and biochemical characteristics of all included participants and when stratified by chronotype preference. In total, $25 \%(\mathrm{n}=159)$ of the cohort identified as morning chronotypes, 52\% ( $\mathrm{n}=330)$ intermediate and 23\% ( $\mathrm{n}=146)$ evening chronotype. Online supplementary table 2 displays the accelerometer derived physical behavior metrics for all included participants and when stratified by chronotype preference. Overall, $759.2 \pm 97.7 \mathrm{~min}$ $(73.4 \%)$ were spent in sedentary behavior, $172.9 \pm 52.1$ min $(24.5 \%)$ in light activity and $14.6(23.7) \min (2.1 \%)$ in MVPA. The average sleep duration was $403.9 \pm 63.9 \mathrm{~min}$. 
Table 1 Participant characteristics of all included individuals and when stratified by chronotype

\begin{tabular}{|c|c|c|c|c|}
\hline & All $(n=635)$ & Morning (n=159) & Intermediate $(n=330)$ & Evening $(n=146)$ \\
\hline \multicolumn{5}{|l|}{ Demographic variables } \\
\hline Age & $63.8 \pm 8.4$ & $64.3 \pm 7.7$ & $64.9 \pm 7.6$ & $60.5 \pm 9.9$ \\
\hline Sex (female) & $220(34.6)$ & 57 (35.8) & $96(29.0)$ & 67 (45.9) \\
\hline Ethnicity (white European) & $534(84.1)$ & $152(95.6)$ & $258(78.2)$ & $124(84.9)$ \\
\hline Current smokers & $35(5.5)$ & $6(3.8)$ & $20(6.1)$ & $9(6.2)$ \\
\hline \multicolumn{5}{|l|}{ Employment status } \\
\hline Employed & $214(33.7)$ & $62(39.0)$ & $100(30.3)$ & $52(35.6)$ \\
\hline Retired & $366(57.6)$ & $89(56.0)$ & 209 (63.3) & $68(46.6)$ \\
\hline Other & $55(8.7)$ & $8(5.0)$ & $21(6.4)$ & $26(17.8)$ \\
\hline \multicolumn{5}{|l|}{ Medication } \\
\hline Insulin & $166(26.1)$ & $32(20.1)$ & $89(27.0)$ & $45(30.8)$ \\
\hline Biguanides & $454(71.5)$ & $114(71.7)$ & $227(68.8)$ & $113(77.4)$ \\
\hline SGLT2i & $56(8.9)$ & $12(7.5)$ & $29(8.8)$ & $15(10.3)$ \\
\hline DPP-4 & $92(14.5)$ & $21(13.2)$ & $48(14.5)$ & $23(15.8)$ \\
\hline GLP-1RA & $36(5.7)$ & $8(5.0)$ & $15(4.5)$ & $13(8.9)$ \\
\hline Sulfonylureas & $138(21.8)$ & $38(23.9)$ & $81(24.5)$ & $19(13.0)$ \\
\hline Lipid lowering & $453(71.4)$ & $76(47.8)$ & $267(80.9)$ & $110(75.3)$ \\
\hline Anti-hypertensive & $427(67.3)$ & $76(47.8)$ & $256(77.6)$ & $95(65.1)$ \\
\hline Duration of diabetes & $11 \pm 8$ & $10.2 \pm 7.5$ & $11.3 \pm 7.9$ & $10.5 \pm 8.2$ \\
\hline Depression & $143(22.5)$ & $39(24.5)$ & $52(15.8)$ & 52 (35.6) \\
\hline \multicolumn{5}{|l|}{ Anthropometric variables } \\
\hline BMI $\left(\mathrm{kg} / \mathrm{m}^{2}\right)$ & $30.9 \pm 5.1$ & $30.5 \pm 5.0$ & $30.6 \pm 4.7$ & $32.3 \pm 5.7$ \\
\hline \multicolumn{5}{|l|}{ Cardiometabolic variables } \\
\hline HbA1c (\%) & $7.1 \pm 1.2$ & $6.9 \pm 1.1$ & $7.1 \pm 1.2$ & $7.2 \pm 1.3$ \\
\hline $\mathrm{HbA} 1 \mathrm{c}(\mathrm{mmol} / \mathrm{mol})$ & $54 \pm 14$ & $52 \pm 12$ & $54 \pm 13$ & $55 \pm 14$ \\
\hline
\end{tabular}

Data presented as mean $\pm \mathrm{SD}$, median (IQR) or number (column percentage).

BMI, body mass index; DPP-4, dipeptidyl-peptidase 4; GLP-1RA, glucagon-like peptide-1 receptor agonists; SGLT2i, sodium-glucose cotransporter 2 inhibitor.

\section{Timing of physical behaviors}

The timing of both sleep onset and physical activity differed across chronotypes (figure 1, online supplementary table 2), with evening chronotypes displaying a later sleep onset and consistently later physical activity

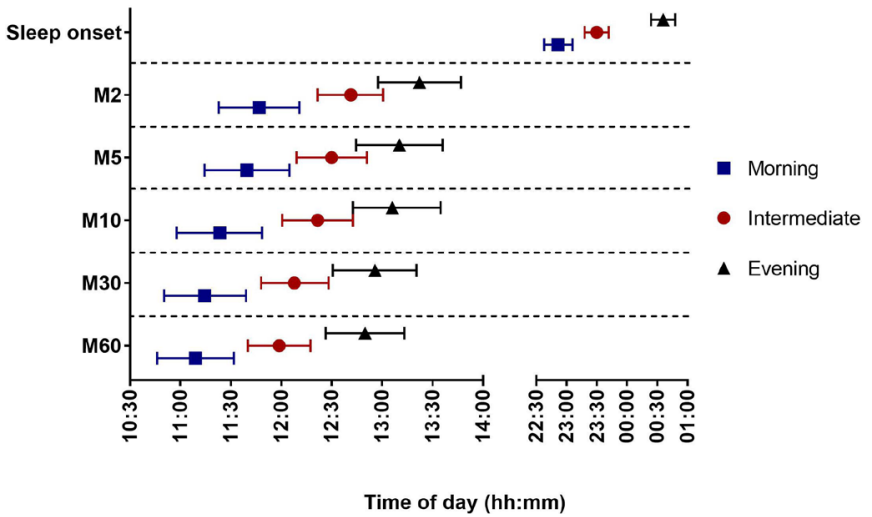

Figure 1 Timing of sleep onset and continuous bouts of physical activity across all chronotypes. time. For example, there was a 1 hour 44 min difference between average sleep onset time for morning (22:52 (22:38 to 23:06)) vs evening chronotypes (00:36 (00:24 to $00: 48)$ ). Similarly, the most active $30 \mathrm{~min}$ of the day (M30 value), on average, occurred 1 hour 42 min earlier in morning (11:14 (10:50 to $11: 39)$ ) vs evening (12:56 (12:31 to 13:20)) chronotypes. These findings reiterate the results of the MEQ.

Table 2 displays the time spent in different physical behaviors by chronotype, with figure 2 displaying the average time spent engaged in sedentary, light or MVPA. The unadjusted values are also presented in online supplementary table 3 . There was no main effect of chronotype on sleep duration.

After adjustment for tertiles of sleep duration, those with an evening chronotype spent 774.7 (754.8 to 794.6) min per day engaged in sedentary time. This level was higher than that for intermediate and morning types which were -37.6 ( -55.0 to -20.3$)$ and -28.7 (-48.3 to -8.6) min per day lower, respectively. Similarly, those 
Table 2 Mean $(95 \% \mathrm{Cl})$ differences in physical behaviors and acceleration variables by chronotype

\begin{tabular}{|c|c|c|c|c|}
\hline & Morning & Intermediate & Evening & $\begin{array}{l}\text { Main } \\
\text { effect for } \\
\text { chronotype }\end{array}$ \\
\hline Sleep duration (min) & 383.8 (369.4 to 398.2 ) & 391.4 (379.3 to 403.4$)$ & 376.1 (361.8 to 390.5$)$ & 0.127 \\
\hline Sedentary time (min) & $746.3(726.1 \text { to } 766.4)^{\star}$ & 737.1 (720.4 to 753.8$) \dagger$ & 774.7 (754.8 to 794.6$)$ & 0.001 \\
\hline Light activity (min) & $189.2(178.0$ to 200.4$) \dagger$ & $181.6(172.4$ to 191.0$) \dagger$ & 155.7 (144.6 to 166.8$)$ & $<0.001$ \\
\hline MVPA (min) & 22.3 (17.1 to 27.5$) \dagger$ & $19.2(14.9 \text { to } 23.5)^{\star}$ & 12.5 (7.4 to 17.7$)$ & 0.001 \\
\hline Daily acceleration (mg) & 23.5 (22.0 to 24.9$) \dagger$ & 22.4 (21.2 to 23.6)† & 19.5 (18.0 to 20.9$)$ & $<0.001$ \\
\hline Continuous M2 (mg) & 169.5 (154.5 to 184.4$) \dagger$ & $156.9(144.5 \text { to } 169.3)^{\star}$ & 140.4 (125.6 to 155.2$)$ & 0.001 \\
\hline Continuous M5 (mg) & $152.2(138.7$ to 165.6$) \dagger$ & $138.7(127.5 \text { to } 149.8)^{\star}$ & 124.5 (111.1 to 137.8$)$ & $<0.001$ \\
\hline Continuous M10 (mg) & 136.5 (124.2 to 148.8$) \dagger$ & $124.4(114.2 \text { to } 134.6)^{\star}$ & 111.5 (99.3 to 123.7$)$ & $<0.001$ \\
\hline Continuous M30 (mg) & 105.2 (94.9 to 115.5$) \dagger$ & $95.5(86.9 \text { to } 104.0)^{*}$ & 84.0 (73.8 to 94.3$)$ & $<0.001$ \\
\hline Continuous M60 (mg) & 83.2 (74.5 to 92.0$) \dagger$ & $76.4(69.2 \text { to } 83.6)^{\star}$ & 67.2 (58.6 to 75.8$)$ & 0.002 \\
\hline Intensity gradient & $-2.73(-2.77 \text { to }-2.69)^{*}$ & $-2.76(-2.79 \text { to }-2.73)^{*}$ & $-2.80(-2.84$ to -2.76$)$ & 0.007 \\
\hline
\end{tabular}

Adjusted for age, sex, ethnicity, employment status, duration of diabetes and sleep duration (tertiles). Tertile cut points were 381.3 min and $432.28 \mathrm{~min}$.

${ }^{*} \mathrm{P}<0.05$ vs evening chronotype.

$\dagger P<0.001$ vs evening chronotype.

MVPA, moderate-to-vigorous physical activity.

with a preference for an evening chronotype spent 155.7 (144.6 to 166.8 ) $\mathrm{min}$ in light intensity physical activity, which was lower than both the intermediate (26.0 (16.3 to 35.7) min per day) and morning (33.5 (22.5 to 44.6) min per day) chronotypes. Evening chronotypes also displayed the lowest levels of MVPA (12.5 (7.4 to 17.7) min per day) after adjustment for covariates. The greatest disparity was observed when compared with morning chronotypes, who engaged in (9.7 (4.6 to 14.9) more MVPA minutes per day ( $56 \%$ difference) $)$.

\section{Average acceleration, MX values and intensity gradient}

Average acceleration was lower in the evening chronotypes (19.5 (18.0 to 20.9) $\mathrm{mg}$ ), when compared with both intermediate (22.4 (21.2 to 23.6) $\mathrm{mg}$ ) and morning chronotypes (23.5 (22.0 to 24.9) $\mathrm{mg}$ ) (both $\mathrm{p}<0.001$ ). The MX metrics (M2, M5, M10, M30, M60) were consistently lower in evening chronotypes compared with both intermediate and morning chronotypes. For example, the M30 value demonstrated that the acceleration for

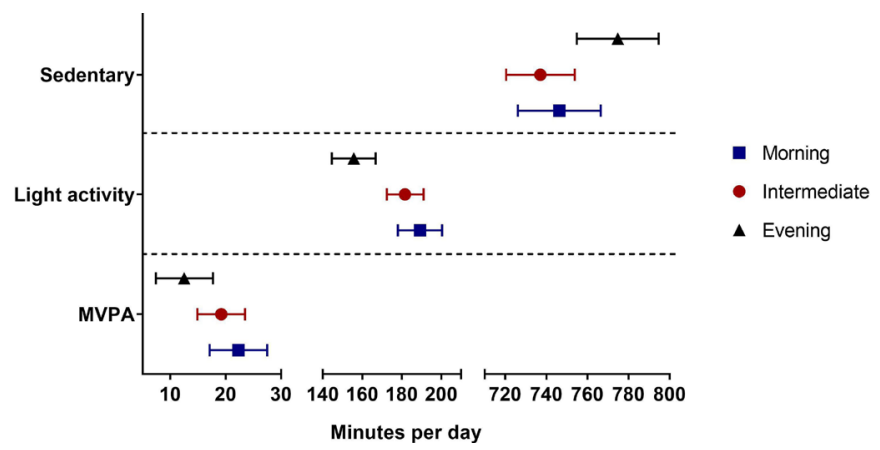

Figure 2 Average time spent engaged in sedentary, light or MVPA across all chronotypes. MVPA, moderate-to-vigorous physical activity. evening chronotypes was $22.4 \%$ lower than morning types (84.0 (73.8 to 94.3 ) vs 105.2 (94.9 to 115.5$) \mathrm{mg}$ ), where an intensity value $\geq 100 \mathrm{mg}$ is equivalent to a slow walk or higher. ${ }^{32}$

Figure 3 presents radar plots illustrating continuous MX metrics for (clockwise) the most active $60 \mathrm{~min}$ (M60), 30 min (M30), 10 min (M10), 5 min (M5) and 2 min (M2) for morning, intermediate and evening chronotypes. The dashed circles show approximate accelerations associated with a slow (blue) and brisk (red) walk. ${ }^{30} 33$ This difference in intensity between chronotypes is also reflected in the intensity gradient value, where those with an evening chronotype $(-2.80$ $(-2.84$ to -2.76$))$ had a lower gradient compared with

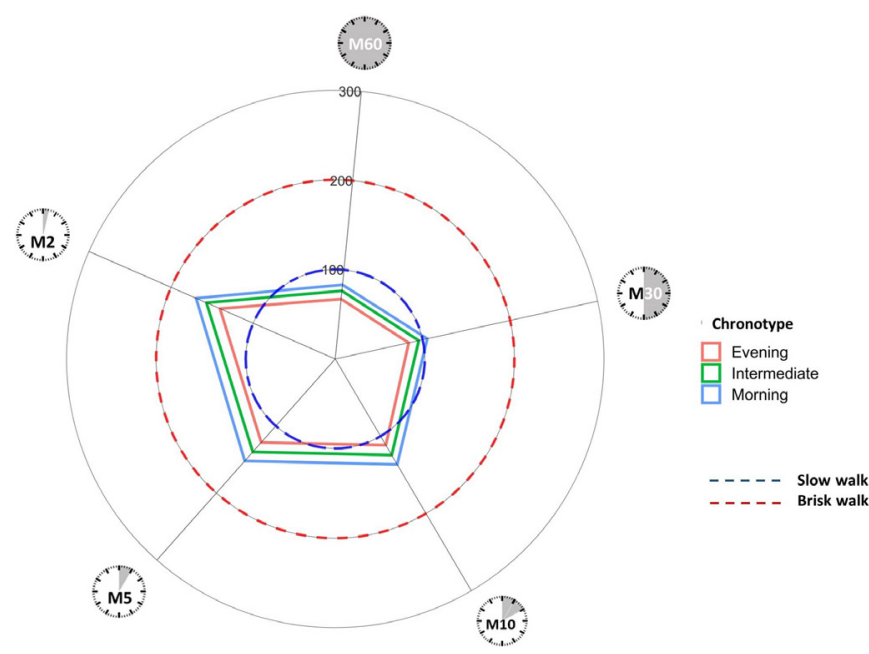

Figure 3 Radar plot illustrating MX metrics (continuous) for (clockwise) the most active $60 \mathrm{~min}$ (M60), $30 \mathrm{~min}$ (M30), 10 min (M10), 5 min (M5) and 2 min (M2) for morning, intermediate and evening chronotypes. 
both morning $(-2.73(-2.77$ to -2.69$))$ and intermediate $(-2.76(-2.79$ to -2.73$))$ types, indicating lower time accumulated at mid-range and higher intensity activities.

There were no significant interactions for ethnicity, age, sex, BMI or employment by chronotype for any of the accelerometer variables (all $\mathrm{p}>0.1$ ) (online supplementary table 4).

\section{CONCLUSION}

This is the first study to investigate the effect of chronotype on device-measured physical behaviors in those with established T2DM. The results demonstrate that, after adjustment for total sleep duration, those with a preference for an evening chronotype had higher sedentary time, lower levels of light activity, lower MVPA and lower mean daily acceleration compared with both morning and intermediate chronotypes. For example, average MVPA was $56 \%$ lower in evening chronotypes compared with morning chronotypes, representing an absolute difference of $10 \mathrm{~min}$. The MX metrics and intensity gradient also suggest that, in addition to duration, differences also exist in the intensity of movement between chronotypes. For example, the M30 value for morning chronotypes was greater than $100 \mathrm{mg}$, which suggests that $30 \mathrm{~min}$ was spent at an intensity equivalent to a slow walk or higher, ${ }^{34}$ whereas the evening chronotypes accumulated their most active $30 \mathrm{~min}$ at a lower intensity $(<100 \mathrm{mg}$, equivalent to 'pottering around'). ${ }^{32}$ Those who reported a preference for an evening chronotype were also confirmed to have a later sleep onset time using accelerometer data. In addition, as well as evening chronotypes having lower levels of physical activity overall, the physical activity that was conducted occurred later on in the day.

The chronotype preference in this study (morning $=25 \%$, intermediate $=52 \%$, evening $=23 \%$ ) is broadly similar to that observed in the general population, although with a slightly larger representation of evening chronotypes in our cohort. For example, a large prospective, population-based cohort study (UK Biobank) demonstrated that $27 \%$ of individuals identified themselves as morning types, $63.9 \%$ as neither/intermediate and $9.1 \%$ as evening types. ${ }^{35}$ The average sleep duration in this cohort (morning $=6.7$ hours, intermediate $=6.8$ hours, evening $=6.6$ hours) is marginally lower ( $\sim 0.5$ hour) than other population based cohort studies that have used accelerometers to quantify sleep duration. ${ }^{36}$ Overall physical activity volume and intensity (MX metrics) were between $20 \%$ and $60 \%$ lower than those reported in office workers or other population based cohorts. ${ }^{33}$ 37-40 Taken together, these collective results indicate that people with T2DM lead a lifestyle characterized by excessive sedentary behaviors and insufficient physical activity volume, with $97.9 \%$ of waking time spent in either sedentary behavior or light physical activity, which may be further exacerbated by an eveningness preference.
Our results also extend those from other studies that have examined both chronotype preference and selfreported physical activity. For example, Wennman et al examined the association between chronotype preference, leisure time physical activity and sitting time in $\sim 5000$ Finnish men and women. ${ }^{41}$ They reported that a self-assessed 'evening type' was typically associated with greater odds of engaging in higher amounts of sitting when compared with 'morning types'. This difference in chronotype was also evident for total physical activity, where evening types had higher odds of engaging in low levels or negligible amounts of physical activity when compared with morning types. ${ }^{41}$ Similarly, a later bedtime and wake time, both evident in this cohort, have been associated with lower levels of device measured, freeliving physical activity (MVPA) in young adults and selfreported physical activity levels in working women. ${ }^{17} 42$

Although the underlying mechanisms are not completely understood, an evening preference may exacerbate the clustering of unhealthy behaviors (eg, physical inactivity), resulting in an increased prevalence of hypertension, ${ }^{10}$ a higher BMI, increased odds of developing $\mathrm{T}_{2} \mathrm{DM}^{9}$ and poorer glycemic control in those with established T2DM. ${ }^{43}$ This was evident in our cohort, as those with an evening chronotype had the highest BMI and HbA1c levels, when compared with morning and intermediate chronotypes. The lower physical activity levels seen in evening chronotypes may also be influenced by social (ie, activities not coinciding with regular social schedules) and physical environment aspects (ie, safety concerns). Moreover, personal/ socially imposed alterations in sleep, as demonstrated by the differences in sleep and physical activity timing, may result in a 'circadian misalignment'. ${ }^{44}$ For example, an enforced early wakeup may reduce the likelihood of engaging in physical activity due to the resulting tiredness or time constraints of family responsibilities in the evening. This may make a natural preference for engaging in physical activity later in the day more difficult to achieve.

Due to its wide-ranging health benefits, minimal cost and side effects and accessibility, physical activity may be an attractive non-pharmacological treatment option that could also theoretically improve circadian misalignment, through alterations in temperature regulation and/or hormone levels. ${ }^{45} 46$ In humans, the circadian clock is divided into two distinct parts, the master clock in the suprachiasmatic nucleus (SCN) of the hypothalamus and peripheral clocks, situated in the peripheral tissues (eg, skeletal muscle). ${ }^{47}$ The peripheral clocks are entrained by the light-dependent regulation of the SCN and by other non-photic zeitgebers (eg, physical activity) for the human circadian system, ${ }^{48}$ thus acting in an SCNindependent manner. ${ }^{49}$ Therefore, in many individuals, especially in people with a late chronotype, an advance of the internal circadian rhythm through informed timing of physical activity may be useful as an adjunct therapeutic strategy to foster chronobiological homeostasis 
and better align internal rhythms with the environment and standard social schedules.

This analysis has strengths and limitations. Most notably, it provides novel evidence in a T2DM population recruited primarily through primary and secondary care using device measured quantification of physical behaviors. The research-grade monitors used to quantify these physical behaviors also allowed for the generation of high resolution raw data, which subsequently facilitated the development of novel activity metrics used in conjunction with more traditional outputs and allowing the physical behaviors to be timestamped. In addition, participants were phenotyped with regard to anthropometric and demographic variables. As such, we were able to investigate and adjust for potential confounders. That said, although cross-sectional data are convenient for hypothesis generation, it does limit the scope of the results and precludes the ability to make causal inference.

In this analysis of 635 participants with T2DM, an evening chronotype was associated with lower physical activity across a range of metrics and higher sedentary time. Overall, there is a need for large-scale interventions to be implemented into diabetes care which support people with T2DM to initiate, maintain and achieve the substantial benefits of an active lifestyle. This may be particular pertinent for those with an 'eveningness' preference, where personalized physical activity interventions may need to place additional emphasis on a whole-day approach, as emphasized by their later sleep onset time and consistently later physical activity time compared with morning and intermediate chronotypes. The focus on low intensity activity is likely to be important in these individuals, as our results suggest that purposeful MVPA is likely to be on the outer reaches of the normal day to day experiences. Even small increases in the percentage of time spent in such activities would be useful toward increasing the volume and distribution of activity.

Acknowledgements We thank Priti Odedra for her continued management and oversight of the study. The authors would also like to thank the participants for taking the time to participate and members of Leicester Diabetes Centre Patient and Public Forum for their involvement in the design of this study. The authors would also like to acknowledge the contribution of the other research sites.

Collaborators CODEC Investigators: Professor Alice C Smith; Dr Carolyn Chee; Professor Frances L Game; Ms Janet Beecham.

Contributors All authors were involved in the study design. JH drafted the manuscript. JH, AVR, CLE and TY contributed to data analysis. All authors contributed to the interpretation of data and provided comments on the final draft of the manuscript. All authors were responsible for the study conception, design, data analysis and drafting. All authors read and approved the final manuscript.

Funding The research was supported by the NIHR Leicester Biomedical Research Centre, which is a partnership between University Hospitals of Leicester NHS Trust, Loughborough University and the University of Leicester.

Competing interests None declared.

Patient consent for publication Not required.

Ethics approval The study received ethical approval from the West Midlands-Black Country Research Ethics Committee (16/WM/0457) and is registered on clinicaltrials. gov (NCT02973412).

Provenance and peer review Not commissioned; externally peer reviewed.
Data availability statement The dataset generated during this study is not publicly available but is available from the corresponding author on reasonable request.

Open access This is an open access article distributed in accordance with the Creative Commons Attribution Non Commercial (CC BY-NC 4.0) license, which permits others to distribute, remix, adapt, build upon this work non-commercially, and license their derivative works on different terms, provided the original work is properly cited, appropriate credit is given, any changes made indicated, and the use is non-commercial. See: http://creativecommons.org/licenses/by-nc/4.0/.

\section{ORCID iDs}

Joseph Henson http://orcid.org/0000-0002-3898-7053

Emma Baldry http://orcid.org/0000-0002-9552-4143

\section{REFERENCES}

1 Kahn SE. The relative contributions of insulin resistance and beta-cell dysfunction to the pathophysiology of type 2 diabetes. Diabetologia 2003;46:3-19.

2 International Diabetes Federation. Diabetes atlas 2019. 9 edn, 2020.

3 Roglic G, Unwin N, Bennett PH, et al. The burden of mortality attributable to diabetes: realistic estimates for the year 2000. Diabetes Care 2005;28:2130-5.

4 Alnaji A, Law GR, Scott EM. The role of sleep duration in diabetes and glucose control. Proc Nutr Soc 2016;75:512-20.

5 Brady EM, Bodicoat DH, Hall AP, et al. Sleep duration, obesity and insulin resistance in a multi-ethnic UK population at high risk of diabetes. Diabetes Res Clin Pract 2018;139:195-202.

6 Smiley A, King D, Bidulescu A. The association between sleep duration and metabolic syndrome: the NHANES 2013/2014. Nutrients 2019;11:11.

7 Duffy JF, Rimmer DW, Czeisler CA. Association of intrinsic circadian period with morningness-eveningness, usual wake time, and circadian phase. Behav Neurosci 2001;115:895-9.

8 Wittmann M, Dinich J, Merrow M, et al. Social jetlag: misalignment of biological and social time. Chronobiol Int 2006;23:497-509.

$9 \mathrm{Yu} \mathrm{JH}$, Yun C-H, Ahn JH, et al. Evening chronotype is associated with metabolic disorders and body composition in middle-aged adults. J Clin Endocrinol Metab 2015;100:1494-502.

10 Merikanto I, Lahti T, Puolijoki H, et al. Associations of chronotype and sleep with cardiovascular diseases and type 2 diabetes. Chronobiol Int 2013;30:470-7.

11 Sluik D, Buijsse B, Muckelbauer R, et al. Physical activity and mortality in individuals with diabetes mellitus: a prospective study and meta-analysis. Arch Intern Med 2012;172:1285-95.

12 Colberg SR, Sigal RJ, Yardley JE, et al. Physical activity/exercise and diabetes: a position statement of the American diabetes association. Diabetes Care 2016;39:2065-79.

13 Office of Disease Prevention and Health Promotion. Physical activity guidlines for Americans. 2 edn, 2019.

14 Cichosz SL, Fleischer J, Hoeyem P, et al. Objective measurements of activity patterns in people with newly diagnosed type 2 diabetes demonstrate a sedentary lifestyle. Diabet Med 2013;30:1063-6.

15 Loprinzi PD. Accelerometer-determined sedentary and physical activity estimates among older adults with diabetes: considerations by demographic and comorbidity characteristics. J Aging Phys Act 2014;22:432-40.

16 Kelly J, Edney K, Moran C, et al. Gender differences in physical activity levels of older people with type 2 diabetes mellitus. $J$ Phys Act Health 2016;13:409-15.

17 Shechter A, St-Onge M-P. Delayed sleep timing is associated with low levels of free-living physical activity in normal sleeping adults. Sleep Med 2014;15:1586-9.

18 Spring B, Ockene JK, Gidding SS, et al. Better population health through behavior change in adults: a call to action. Circulation 2013;128:2169-76.

19 Brady EM, Hall AP, Baldry E, et al. Rationale and design of a cross-sectional study to investigate and describe the chronotype of patients with type 2 diabetes and the effect on glycaemic control: the CODEC study. BMJ Open 2019;9:e027773:2018-7773.

20 Horne JA, Ostberg O. A self-assessment questionnaire to determine morningness-eveningness in human circadian rhythms. Int $J$ Chronobiol 1976;4:97-110.

21 Taillard J, Philip P, Chastang J-F, et al. Validation of horne and ostberg morningness-eveningness questionnaire in a middle-aged population of French workers. J Biol Rhythms 2004;19:76-86.

22 van Hees VT, Gorzelniak L, Dean León EC, et al. Separating movement and gravity components in an acceleration signal and 
implications for the assessment of human daily physical activity. PLoS One 2013;8:e61691.

23 van Hees VT, Fang Z, Langford J, et al. Autocalibration of accelerometer data for free-living physical activity assessment using local gravity and temperature: an evaluation on four continents. J Appl Physiol 2014;117:738-44.

24 Migueles JH, Rowlands AV, Huber F, et al. GGIR: a research community-driven open source $R$ package for generating physical activity and sleep outcomes from multi-day RAW accelerometer data. J Meas Phys Behav 2019;2:188-96.

25 van Hees VT, Sabia S, Jones SE, et al. Estimating sleep parameters using an accelerometer without sleep diary. Sci Rep 2018;8:12975.

26 Rowlands AV, Mirkes EM, Yates T, et al. Accelerometer-assessed physical activity in epidemiology: are monitors equivalent? Med Sci Sports Exerc 2018;50:257-65.

27 Bakrania K, Yates T, Rowlands AV, et al. Intensity thresholds on RAW acceleration data: Euclidean norm minus one (ENMO) and mean amplitude deviation (Mad) approaches. PLoS One 2016;11:e0164045.

28 Hildebrand M, Hansen BH, van Hees VT, et al. Evaluation of raw acceleration sedentary thresholds in children and adults. Scand J Med Sci Sports 2017;27:1814-23.

29 Menai M, van Hees VT, Elbaz A, et al. Accelerometer assessed moderate-to-vigorous physical activity and successful ageing: results from the Whitehall II study. Sci Rep 2017;8:45772.

30 Rowlands AV, Fairclough SJ, Yates T, et al. Activity intensity, volume, and norms: utility and interpretation of Accelerometer metrics. Med Sci Sports Exerc 2019;51:2410-22.

31 Rowlands AV, Edwardson CL, Davies MJ, et al. Beyond cut points: accelerometer metrics that capture the physical activity profile. Med Sci Sports Exerc 2018;50:1323-32.

32 Rowlands AV, Dawkins NP, Maylor B, et al. Enhancing the value of accelerometer-assessed physical activity: meaningful visual comparisons of data-driven translational accelerometer metrics. Sports Med Open 2019;5:47.

33 Rowlands AV, Sherar LB, Fairclough SJ, et al. A data-driven, meaningful, easy to interpret, standardised accelerometer outcome variable for global surveillance. J Sci Med Sport 2019;22:1132-1138.

34 Hildebrand M, VAN Hees VT, Hansen BH, et al. Age group comparability of raw accelerometer output from wrist- and hip-worn monitors. Med Sci Sports Exerc 2014;46:1816-24.

35 Patterson F, Malone SK, Lozano A, et al. Smoking, screen-based sedentary behavior, and diet associated with habitual sleep duration and Chronotype: data from the UK Biobank. Ann Behav Med 2016;50:715-26.

36 Dashti HS, Jones SE, Wood AR, et al. Genome-Wide association study identifies genetic loci for self-reported habitual sleep duration supported by accelerometer-derived estimates. Nat Commun 2019;10:1100.

37 Edwardson CL, Biddle SJH, Clarke-Cornwell A, et al. A three arm cluster randomised controlled trial to test the effectiveness and costeffectiveness of the SMART Work \& Life intervention for reducing daily sitting time in office workers: study protocol. BMC Public Health 2018;18:1120.

38 Cassidy S, Fuller H, Chau J, et al. Accelerometer-derived physical activity in those with cardio-metabolic disease compared to healthy adults: a UK Biobank study of 52,556 participants. Acta Diabetol 2018;55:975-9.

39 Doherty A, Jackson D, Hammerla N, et al. Large scale population assessment of physical activity using wrist worn Accelerometers: the UK Biobank study. PLoS One 2017;12:e0169649.

40 Stiles VH, Metcalf BS, Knapp KM, et al. A small amount of precisely measured high-intensity habitual physical activity predicts bone health in pre- and post-menopausal women in UK Biobank. Int $J$ Epidemiol 2017;46:1847-56.

41 Wennman $\mathrm{H}$, Kronholm E, Partonen T, et al. Evening typology and morning tiredness associates with low leisure time physical activity and high sitting. Chronobiol Int 2015;32:1090-100.

42 Haraszti Réka Ágnes, Purebl G, Salavecz G, et al. Morningnesseveningness interferes with perceived health, physical activity, diet and stress levels in working women: a cross-sectional study. Chronobiol Int 2014;31:829-37.

43 Reutrakul S, Hood MM, Crowley SJ, et al. Chronotype is independently associated with glycemic control in type 2 diabetes. Diabetes Care 2013;36:2523-9.

44 Arora T, Taheri S. Associations among late chronotype, body mass index and dietary behaviors in young adolescents. Int $\mathrm{J}$ Obes 2015;39:39-44.

45 Waterhouse J, Drust B, Weinert D, et al. The circadian rhythm of core temperature: origin and some implications for exercise performance. Chronobiol Int 2005;22:207-25.

46 Hower IM, Harper SA, Buford TW, et al. Exercise, and cardiovascular health. J Circadian Rhythms 2018;16:7.

47 Brown AJ, Pendergast JS, Yamazaki S, et al. Peripheral circadian oscillators. Yale J Biol Med 2019;92:327-35.

48 Lewis P, Korf HW, Kuffer L, et al. Exercise time cues (zeitgebers) for human circadian systems can foster health and improve performance: a systematic review. BMJ Open Sport Exerc Med 2018;4:e000443.

49 Aoyama S, Shibata S. The role of circadian rhythms in muscular and osseous physiology and their regulation by nutrition and exercise. Front Neurosci 2017;11:63. 